\title{
The Effect of Collector in Solar Still for Water Productivity Using Runge-Kutta Method
}

\author{
Mardlijah, Achmad Fatoni, and Lukman Hanafi
}

\begin{abstract}
Solar still is a renewable energy technology. It can reduce crisis of clean and healthy water in some countries. Solar still produces clean and healthy water using the sunlight, but the result of distillate water is not so much. Hence the need for modifications with the addition of collector can increase the yield of distillate water. Mathematical model of solar still with collector is in the form of system of differential equations. It can be solved numerically using Runge-Kutta method. From the simulation, we conclude that the collector increases the amount of distillate water in the solar still.
\end{abstract}

Index Terms-Runge-Kutta method, solar still.

\section{INTRODUCTION}

W ATER is the most important thing in daily life. The consumed water must be clean and healthy water. However the existence of clean and healthy water in the world are limited. More than $2 / 3$ of world surface covered by water and more than $97 \%$ water in the world is brine and about $2.6 \%$ is freshwater but only $1 \%$ of total freshwater can be consumed [1]. The amount of consumed water are limited while the human population is increased. Thus we need a technology that can increase water production.

Solar still is a technology to increase the production of clean and healthy water using sunlight. Unfortunately the amount of produced water produced is not so much. Malik et. al. [2] stated that conventionally solar still produces only few clean and healthy water so that it is necessary to increase the resulting water. Phadatare et. al. [3] has developed an approach by using deepen water in basin. From his research, the result of distillate water is not significant. Then Dwivedi et. al. [4] add two inclined mirrors as solar still cover to catch radiation and distiller. Tanaka [5] research on the influence of angle change to cover mirror in solar still. However the results of these researches are still not sufficient.

In this paper, we add collector in solar still. The collector will increase water temperature in the basin so that both the evaporation process and the amount water are increased. The mathematical model is in the form of system of differential equations. We solve the model numerically by using the Runge-Kutta method.

\section{Mathematical Model}

The modification of solar still by adding collector is shown below:

Manuscript received January 5, 2016; accepted January 20, 2016.

The authors are with the Department of Mathematics, Institut Teknologi Sepuluh Nopember, Surabaya 60111, Indonesia. Emails: \{mardlijah, achmad11, lukman\}@matematika.its.ac.id

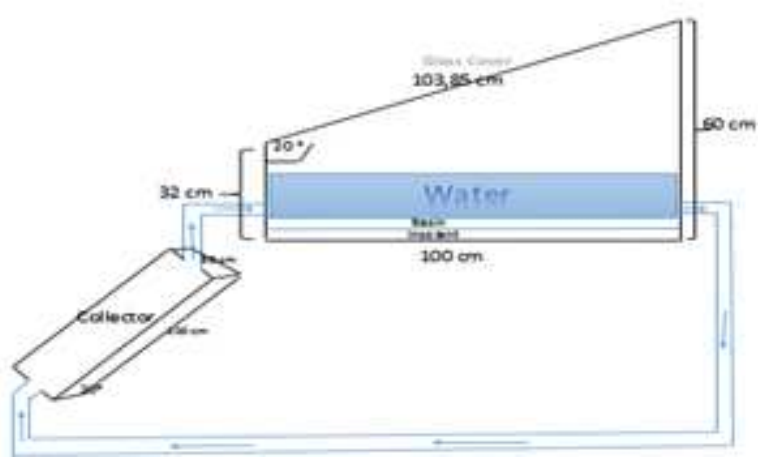

Fig. 1. Solar still with collector.

In Fig. 1, the function of collector is to catch the sunlight radiation. Then the radiation is channeled to water through pipe to basin. The sunlight radiation causes the water temperature to increase. When the water goes back to basin, it will add the evaporation process, which produces more water.

The model of heat balance in solar still with collector is given by Karroute [6] as follows :

$$
\begin{aligned}
& Q_{r, g, a}+Q_{c, g, a}+\frac{m_{g} C_{g}}{2} \frac{d T_{g o}}{d t}=Q_{c d, g}+I_{g} \\
& Q_{r, g, w}+Q_{c, g, w}+Q_{e}+\frac{m_{g} C_{g}}{2} \frac{d T_{g i}}{d t}=Q_{c d, g}+I_{g} \\
& Q_{r, g, w}+Q_{c, g, w}+Q_{e}+m_{w} C_{w} \frac{d T_{w}}{d t}+m_{e} C_{w}\left(T_{w}-T_{a}\right) \\
& \quad=Q_{c o l l}+Q_{c, w, b}+I_{w} \\
& Q_{c, w, b}+Q_{c d, b}+m_{b} C_{b} \frac{d T_{b}}{d t}=I_{b} \\
& Q_{c d, i}+\frac{m_{i} C_{i}}{2} \frac{d T_{i i}}{d t}=Q_{c d, b} \\
& Q_{r, i, a}+Q_{c, i, a}+\frac{m_{i} C_{i}}{2} \frac{d T_{i o}}{d t}=Q_{c d, i}
\end{aligned}
$$

From this model and by substituting the heat transfer as conduction, convection and radiation, we obtain the following results.

1) Heat balance outside mirror

$$
\begin{aligned}
& \frac{d T_{g o}}{d t}=\frac{2}{m_{g} C_{g}}\left(I_{g}+\frac{A_{g} k_{g}}{\Delta l_{g}}\left(T_{g i}-T_{g o}\right)\right. \\
& \left.\quad-A_{g} \in_{g} \sigma\left(T_{g o}^{4}-T_{a}^{4}\right)-A_{g} h_{c, g, a}\left(T_{g o}-T_{a}\right)\right)
\end{aligned}
$$

2) Heat balance inside mirror

$$
\begin{aligned}
& \frac{d T_{g i}}{d t}=I_{g}+A_{g} \frac{A_{g} k_{g}}{\Delta l_{g}}\left(T_{g o}-T_{g i}\right) \\
& \quad-A_{g} \in_{g} \sigma\left(T_{g i}^{4}-T_{w}^{4}\right)-A_{g} h_{c, g, w}\left(T_{g i}-T_{w}\right)-Q_{e}
\end{aligned}
$$


3) Heat transfer in water

$$
\begin{aligned}
& \frac{d T_{w}}{d t}=\frac{1}{m_{w} C_{w}}\left(Q_{\text {coll }}+I_{w}+A_{w} h_{c, w, b}\left(T_{w}-T_{b}\right)\right. \\
& \quad-A_{w} \in_{w} \sigma\left(T_{w}^{4}-T_{g i}^{4}\right)-A_{w} h_{c, g, w}\left(T_{w}-T_{g i}\right) \\
& \left.-Q_{e}-m_{e} C_{w}\left(T_{w}-T_{a}\right)\right)
\end{aligned}
$$

4) Heat transfer in basin

$$
\begin{aligned}
& \frac{d T_{b}}{d t}=\frac{1}{m_{b} C_{b}}\left(I_{b}-A_{b} h_{c, b, w}\left(T_{b}-T_{w}\right)\right. \\
& \left.\quad-\frac{A_{b} k_{b}}{\Delta l_{b}}\left(T_{i i}-T_{b}\right)\right)
\end{aligned}
$$

5) Heat transfer inside insolant

$$
\frac{d T_{i i}}{d t}=\frac{2}{m_{i} C_{i}}\left(\frac{A_{i} k_{i}}{\Delta l_{i}}\left(T_{b}-T_{i o}\right)\right)
$$

6) Heat transfer outside insolant

$$
\begin{aligned}
& \frac{d T_{i o}}{d t}=\frac{2}{m_{i} C_{i}}\left(\frac{A_{i} k_{i}}{\Delta l_{i}}\left(T_{i i}-T_{i o}\right)\right. \\
& \left.\quad-A_{i} \in_{i} \sigma\left(T_{i o}^{4}-T_{a}^{4}\right)-A_{i} h_{c, i, a}\left(T_{i o}-T_{a}\right)\right)
\end{aligned}
$$

$Q_{e}$ obtained by Zejli [7]:

$$
Q_{e}=A_{w} h_{e}\left(T_{w}-T_{g i}\right)
$$

$h_{e}$ obtained by Dev [8]:

$$
h_{e}=16273 \times 10^{-3} h_{c, g, w} \frac{P_{w}-P_{g i}}{T_{w}-T_{g i}}
$$

$h_{c, g, w}$ obtained by Phadatare [3]:

$h_{c, g, w}=0.884\left[\left(T_{w}-T_{g i}\right)+\frac{\left(P_{w}-P_{g i}\right)\left(T_{w}-T_{g i}\right)}{2689 \times 10^{3}-P_{w}}\right]$

$P_{w}$ and $P_{g i}$ obtained by Sampathkumar [9]:

$$
\begin{aligned}
P_{w} & =\exp \left[25.317-\frac{5144}{T_{w}+273}\right] \\
P_{g i} & =\exp \left[25.317-\frac{5144}{T_{g i}+273}\right]
\end{aligned}
$$

$m_{e}$ obtained by Dwivedi [4]:

$$
m_{e}=\frac{h_{e}\left(T_{w}-T_{g i}\right) 3600}{L_{v}}
$$

where

$$
L_{v}=3408-5.21 T_{a}+0.01 T_{a}^{2}-1.194 T_{a}^{3}
$$

and

$$
I_{w}=I_{d i r}+I_{d i f f}
$$

$Q_{\text {coll }}$ obtained by Dimri [10]:

$$
Q_{\text {coll }}=A_{\text {coll }}\left(F_{R}\left[(\alpha \tau)_{\text {coll }}\right] I_{\text {coll }}-U_{L, \text { coll }}\left(T_{w}-T_{a}\right)\right)
$$

\section{NUMERICAL SOLUTION}

We use the Runge-Kutta method to obtain the solution of the model numerically, as follows.

$$
\begin{array}{r}
T g o_{n+1}=T g o_{n}+\frac{1}{6}\left(k_{1, T g o}+2 k_{2, T g o}+2 k_{3, T g o}+k_{4, T g o}\right) \\
T g i_{n+1}=T g i_{n}+\frac{1}{6}\left(k_{1, T g i}+2 k_{2, T g i}+2 k_{3, T g i}+k_{4, T g i}\right) \\
T w_{n+1}=T w_{n}+\frac{1}{6}\left(k_{1, T w}+2 k_{2, T w}+2 k_{3, T w}+k_{4, T w}\right) \\
T b_{n+1}=T b_{n}+\frac{1}{6}\left(k_{1, T b}+2 k_{2, T b}+2 k_{3, T b}+k_{4, T b}\right) \\
T i i_{n+1}=T i i_{n}+\frac{1}{6}\left(k_{1, T i i}+2 k_{2, T i i}+2 k_{3, T i i}+k_{4, T i i}\right) \\
T i o_{n+1}=T i o_{n}+\frac{1}{6}\left(k_{1, T i o}+2 k_{2, T i o}+2 k_{3, T i o}+k_{4, T i o}\right)
\end{array}
$$

where

$$
\begin{array}{r}
k_{1, T g o}=h f\left(t_{0}, T g o_{0}, T g i_{0}, T w_{0}, T b_{0}, T i i_{0}, T i o_{0}\right) \\
k_{1, T g i}=h f\left(t_{0}, T g o_{0}, T g i_{0}, T w_{0}, T b_{0}, T i i_{0}, T i o_{0}\right) \\
k_{1, T w}=h f\left(t_{0}, T g o_{0}, T g i_{0}, T w_{0}, T b_{0}, T i i_{0}, T i o_{0}\right) \\
k_{1, T b}=h f\left(t_{0}, T g o_{0}, T g i_{0}, T w_{0}, T b_{0}, T i i_{0}, T i o_{0}\right) \\
k_{1, T i i}=h f\left(t_{0}, T g o_{0}, T g i_{0}, T w_{0}, T b_{0}, T i i_{0}, T i o_{0}\right) \\
k_{1, T i o}=h f\left(t_{0}, T g o_{0}, T g i_{0}, T w_{0}, T b_{0}, T i i_{0}, T i o_{0}\right) \\
k_{2, T g o}=h f\left(t_{0}+\frac{h}{2}, T g o_{0}+\frac{k_{1, T g o}}{2}, T g i_{0}+\frac{k_{1, T g i}}{2},\right.
\end{array}
$$$$
\left.T w_{0}+\frac{k_{1, T w}}{2}, T b_{0}+\frac{k_{1, T b}}{2}, T i i_{0}+\frac{k_{1, T i i}}{2}, T i o_{0}+\frac{k_{1, T i o}}{2}\right)
$$

$$
k_{2, T g i}=h f\left(t_{0}+\frac{h}{2}, T g o_{0}+\frac{k_{1, T g o}}{2}, T g i_{0}+\frac{k_{1, T g i}}{2},\right.
$$

$\left.T w_{0}+\frac{k_{1, T w}}{2}, T b_{0}+\frac{k_{1, T b}}{2}, T i i_{0}+\frac{k_{1, T i i}}{2}, T i o_{0}+\frac{k_{1, T i o}}{2}\right)$

$$
k_{2, T w}=h f\left(t_{0}+\frac{h}{2}, T g o_{0}+\frac{k_{1, T g o}}{2}, T g i_{0}+\frac{k_{1, T g i}}{2},\right.
$$

$\left.T w_{0}+\frac{k_{1, T w}}{2}, T b_{0}+\frac{k_{1, T b}}{2}, T i i_{0}+\frac{k_{1, T i i}}{2}, T i o_{0}+\frac{k_{1, T i o}}{2}\right)$ $k_{2, T b}=h f\left(t_{0}+\frac{h}{2}, T g o_{0}+\frac{k_{1, T g o}}{2}, T g i_{0}+\frac{k_{1, T g i}}{2}\right.$,

$\left.T w_{0}+\frac{k_{1, T w}}{2}, T b_{0}+\frac{k_{1, T b}}{2}, T i i_{0}+\frac{k_{1, T i i}}{2}, T i o_{0}+\frac{k_{1, T i o}}{2}\right)$

$$
k_{2, T i i}=h f\left(t_{0}+\frac{h}{2}, T g o_{0}+\frac{k_{1, T g o}}{2}, T g i_{0}+\frac{k_{1, T g i}}{2},\right.
$$

$\left.T w_{0}+\frac{k_{1, T w}}{2}, T b_{0}+\frac{k_{1, T b}}{2}, T i i_{0}+\frac{k_{1, T i i}}{2}, T i o_{0}+\frac{k_{1, T i o}}{2}\right)$ $k_{2, T i o}=h f\left(t_{0}+\frac{h}{2}, T g o_{0}+\frac{k_{1, T g o}}{2}, T g i_{0}+\frac{k_{1, T g i}}{2}\right.$, $\left.T w_{0}+\frac{k_{1, T w}}{2}, T b_{0}+\frac{k_{1, T b}}{2}, T i i_{0}+\frac{k_{1, T i i}}{2}, T i o_{0}+\frac{k_{1, T i o}}{2}\right)$ $k_{3, T g o}=h f\left(t_{0}+\frac{h}{2}, T g o_{0}+\frac{k_{2, T g o}}{2}, T g i_{0}+\frac{k_{2, T g i}}{2}\right.$, $\left.T w_{0}+\frac{k_{2, T w}}{2}, T b_{0}+\frac{k_{2, T b}}{2}, T i i_{0}+\frac{k_{2, T i i}}{2}, T i o_{0}+\frac{k_{2, T i o}}{2}\right)$ $k_{3, T g i}=h f\left(t_{0}+\frac{h}{2}, T g o_{0}+\frac{k_{2, T g o}}{2}, T g i_{0}+\frac{k_{2, T g i}}{2}\right.$, 


$$
\begin{aligned}
& \left.T w_{0}+\frac{k_{2, T w}}{2}, T b_{0}+\frac{k_{2, T b}}{2}, T i i_{0}+\frac{k_{2, T i i}}{2}, T i o_{0}+\frac{k_{2, T i o}}{2}\right) \\
& k_{3, T w}=h f\left(t_{0}+\frac{h}{2}, T g o_{0}+\frac{k_{2, T g o}}{2}, T g i_{0}+\frac{k_{2, T g i}}{2},\right. \\
& \left.T w_{0}+\frac{k_{2, T w}}{2}, T b_{0}+\frac{k_{2, T b}}{2}, T i i_{0}+\frac{k_{2, T i i}}{2}, T i o_{0}+\frac{k_{2, T i o}}{2}\right) \\
& k_{3, T b}=h f\left(t_{0}+\frac{h}{2}, T g o_{0}+\frac{k_{2, T g o}}{2}, T g i_{0}+\frac{k_{2, T g i}}{2},\right. \\
& \left.T w_{0}+\frac{k_{2, T w}}{2}, T b_{0}+\frac{k_{2, T b}}{2}, T i i_{0}+\frac{k_{2, T i i}}{2}, T i o_{0}+\frac{k_{2, T i o}}{2}\right) \\
& k_{3, T i i}=h f\left(t_{0}+\frac{h}{2}, T g o_{0}+\frac{k_{2, T g o}}{2}, T g i_{0}+\frac{k_{2, T g i}}{2},\right. \\
& \left.T w_{0}+\frac{k_{2, T w}}{2}, T b_{0}+\frac{k_{2, T b}}{2}, T i i_{0}+\frac{k_{2, T i i}}{2}, T i o_{0}+\frac{k_{2, T i o}}{2}\right) \\
& k_{3, T i o}=h f\left(t_{0}+\frac{h}{2}, T g o_{0}+\frac{k_{2, T g o}}{2}, T g i_{0}+\frac{k_{2, T g i}}{2},\right. \\
& \left.T w_{0}+\frac{k_{2, T w}}{2}, T b_{0}+\frac{k_{2, T b}}{2}, T i i_{0}+\frac{k_{2, T i i}}{2}, T i o_{0}+\frac{k_{2, T i o}}{2}\right) \\
& k_{4, T g o}=h f\left(t_{0}+h, T g o_{0}+k_{3, T g o}, T g i_{0}+k_{3, T g i},\right. \\
& \left.T w_{0}+k_{3, T w}, T b_{0}+k_{3, T b}, T i i_{0}+k_{3, T i i}, T i o_{0}+k_{3, T i o}\right) \\
& k_{4, T g i}=h f\left(t_{0}+h, T g o_{0}+k_{3, T g o}, T g i_{0}+k_{3, T g i},\right. \\
& \left.T w_{0}+k_{3, T w}, T b_{0}+k_{3, T b}, T i i_{0}+k_{3, T i i}, T i o_{0}+k_{3, T i o}\right) \\
& k_{4, T w}=h f\left(t_{0}+h, T g o_{0}+k_{3, T g o}, T g i_{0}+k_{3, T g i},\right. \\
& \left.T w_{0}+k_{3, T w}, T b_{0}+k_{3, T b}, T i i_{0}+k_{3, T i i}, T i o_{0}+k_{3, T i o}\right) \\
& k_{4, T b}=h f\left(t_{0}+h, T g o_{0}+k_{3, T g o}, T g i_{0}+k_{3, T g i},\right. \\
& \left.T w_{0}+k_{3, T w}, T b_{0}+k_{3, T b}, T i i_{0}+k_{3, T i i}, T i o_{0}+k_{3, T i o}\right) \\
& k_{4, T i i}=h f\left(t_{0}+h, T g o_{0}+k_{3, T g o}, T g i_{0}+k_{3, T g i},\right. \\
& \left.T w_{0}+k_{3, T w}, T b_{0}+k_{3, T b}, T i i_{0}+k_{3, T i i}, T i o_{0}+k_{3, T i o}\right) \\
& k_{4, T i o}=h f\left(t_{0}+h, T g o_{0}+k_{3, T g o}, T g i_{0}+k_{3, T g i},\right. \\
& \left.T w_{0}+k_{3, T w}, T b_{0}+k_{3, T b}, T i i_{0}+k_{3, T i i}, T i o_{0}+k_{3, T i o}\right)
\end{aligned}
$$

The computation uses the initial temperature and parameter values shown in Table I.

TABLE I

INITIAL TEMPERATURE IN SOLAR STILL.

\begin{tabular}{|c|c|}
\hline Variable & Initial temperature \\
\hline Tgo & $28^{\circ} \mathrm{C}$ \\
Tgi & $28^{\circ} \mathrm{C}$ \\
Tw & $31^{\circ} \mathrm{C}$ \\
Tb & $33^{\circ} \mathrm{C}$ \\
Tii & $31^{\circ} \mathrm{C}$ \\
Tio & $31^{\circ} \mathrm{C}$ \\
\hline
\end{tabular}

Coefficient of convection from water to basin and coefficient of convection from insolent to ambient air are:

$$
\begin{array}{r}
h_{c, w, b}=842.573706\left(T_{b}-T_{w}\right)^{0.25} \\
h_{c, i, a}=2.89267\left(T_{a}-T_{i o}\right)^{0.25}
\end{array}
$$

The simulation results are shown in the following figures.

From Fig. 2, we conclude that the water temperature for solar still without collector in 12 hours (i.e. during observation) reaches $32.25^{\circ} \mathrm{C}$, while solar still with collector reaches $32.8^{\circ} \mathrm{C}$.
TABLE II

PARAMETER VALUES IN SOLAR STILL BY ADDING COLLECTOR [2], [7], [10].

\begin{tabular}{|c|c|c|c|}
\hline Parameter & Value & Parameter & Value \\
\hline$m_{g} C_{g}$ & $6749.29416 \mathrm{j} /{ }^{\circ} \mathrm{C}$ & $k_{g}$ & $0.84 \mathrm{j} / \mathrm{m}^{\circ} \mathrm{C}$ \\
$m_{w} C_{w}$ & $83760 \mathrm{j} /{ }^{\circ} \mathrm{C}$ & $k_{b}$ & $0.3463 \mathrm{j} / \mathrm{m}^{\circ} \mathrm{C}$ \\
$m_{b} C_{b}$ & $12244.68 \mathrm{j} /{ }^{\circ} \mathrm{C}$ & $k_{i}$ & $0.04 \mathrm{j} / \mathrm{m}^{\circ} \mathrm{C}$ \\
$m_{i} C_{i}$ & $5596.5 \mathrm{j} /{ }^{\circ} \mathrm{C}$ & $\epsilon_{g}$ & 0.94 \\
$I_{g}$ & $29.6 \mathrm{w} / \mathrm{m}^{2}$ & $\epsilon_{w}$ & 0.95 \\
$I_{\text {dir }}$ & $592 \mathrm{w} / \mathrm{m}^{2}$ & $\epsilon_{i}$ & 0.885 \\
$I_{b}$ & $473.6 \mathrm{w} / \mathrm{m}^{2}$ & $\sigma$ & $5.67 \times 10^{-8} \mathrm{w} / \mathrm{m}^{2} \mathrm{~K}^{4}$ \\
$I_{\text {diff }}$ & $355.2 \mathrm{w} / \mathrm{m}^{2}$ & $h_{c, g, a}$ & $22.71 \mathrm{w} / \mathrm{m}^{\circ}{ }^{\circ} \mathrm{C}$ \\
$I_{\text {coll }}$ & $473.6 \mathrm{w} / \mathrm{m}^{2}$ & $h_{c, b, w}$ & $137.373 \mathrm{w} / \mathrm{m}^{2}{ }^{\circ} \mathrm{C}$ \\
$A_{g}$ & $1 \mathrm{~m}^{2}$ & $h_{c, i, a}$ & $5.138523\left(T_{a}-T_{i o}\right)^{0.25}$ \\
$A_{w}$ & $1 \mathrm{~m}^{2}$ & $h_{c, w, b}$ & $842.573706\left(T_{b}-T_{w}\right)^{0.25}$ \\
$A_{b}$ & $1 \mathrm{~m}^{2}$ & $F_{R}$ & 0.8 \\
$A_{i}$ & $2.84 \mathrm{~m}^{2}$ & $(\alpha \tau)_{\text {coll }}$ & 0.8 \\
$A_{\text {coll }}$ & $2 \mathrm{~m}^{2}$ & $U_{L, \text { coll }}$ & $6 \mathrm{w} / \mathrm{m}^{2}{ }^{\circ} \mathrm{C}$ \\
$\Delta l_{g}$ & $0.003 \mathrm{~m}^{2}$ & $\Delta l_{i}$ & $0.005 \mathrm{~m}^{2}$ \\
$\Delta l_{b}$ & $0.005 \mathrm{~m}^{2}$ & $T_{a}$ & $32.8^{\circ} \mathrm{C}$ \\
\hline
\end{tabular}

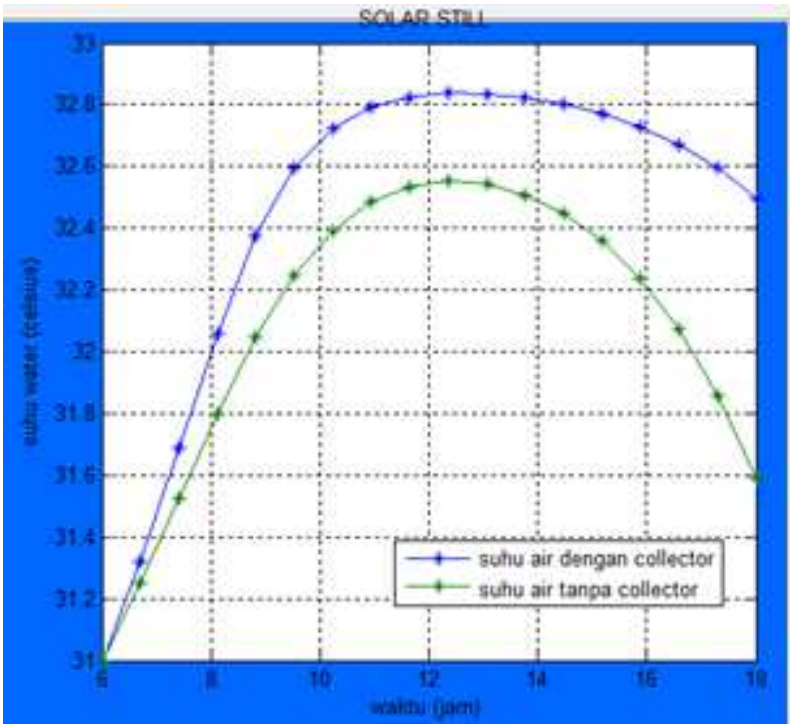

Fig. 2. Comparison of water temperature in solar still between without collector and with collector.

From Fig. 3, we conclude that solar still without collector has a maximum evaporation coefficient not more than $5 \mathrm{w} / \mathrm{m}^{2 \circ}$ while solar still with collector has $6.4 \mathrm{w} / \mathrm{m}^{2 \circ}$ as its maximum.

From Fig. 4, we conclude that the result still from solar still with collector is higher than the solar still without collector.

\section{CONCLusions}

From the results in the previous section, we have the following conclusions:

- Adding collector in solar still will increase the water temperature. Furthermore the coefficient of evaporation is also increased. It follows that the evaporation process will increase as well.

- The result still in solar still without collector produces 4.54109 litres in 12 hours, while solar still with collector produces 8.8289 litres. 


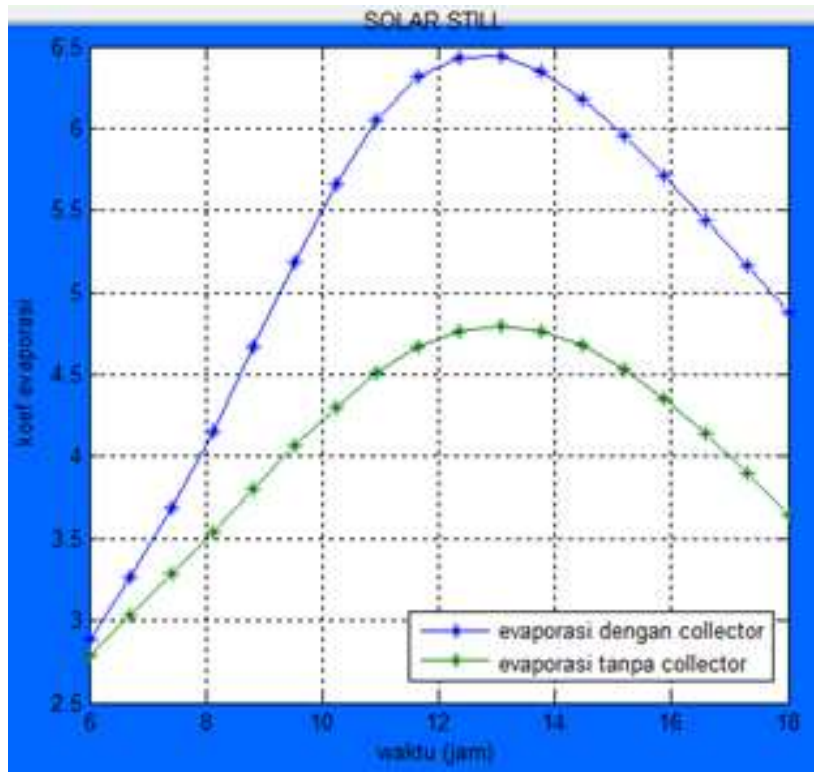

Fig. 3. Comparison of evaporation coefficient between without collector and with collector.

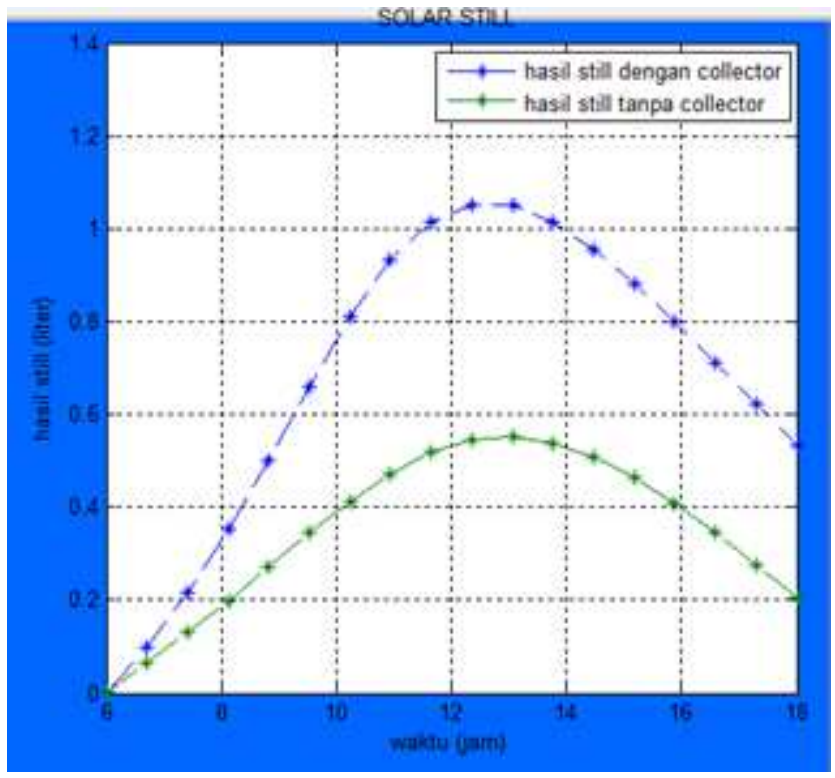

Fig. 4. Comparison of still result between without collector and with collector.

\section{REFERENCES}

[1] N. S. Gokilavani, D. Prabhakaran, and T. Kannadasan, "Various models of traditional solar distillation system for water desalination-a review," Journal of Chemical, Biological and Physical Sciences (JCBPS), vol. 4, no. 2, pp. 1419-1424, 2014.

[2] M. Malik, G. Tiwari, A. Kumar, and M. Sodha, Solar Distillation. Oxford, UK: Pergamon press, 1982.

[3] M. Phadatare and S. Verma, "Influence of water depth on internal heat and mass transfer in a plastic solar still," Desalination, vol. 217, no. 1-3, pp. 267-275, 2007.

[4] V. Dwivedi and G. Tiwari, "Experimental validation of thermal model of a double slope active solar still under natural circulation mode," Desalination, vol. 250, no. 1, pp. 49-55, 2010.

[5] H. Tanaka, "Monthly optimum inclination of glass cover and external reflector of a basin type solar still with internal and external reflector," Solar Energy, vol. 84, no. 11, pp. 1959-1966, 2010.

[6] S. Karroute and A. Chaker, "Theoretical and numerical study of the effect of coupling a collector and reflector on the solar still effiency," in 5th International Renewable Energy Congress (IREC), Mar. 2014, pp. $1-5$.

[7] D. Zejli, R. Benchrifa, A. Bennouna, and O. Bouhelal, "A solar adsorption desalination device: first simulation results," Desalination, vol. 168, pp. 127-135, 2004.

[8] R. Dev and G. Tiwari, "Characteristic equation of a passive solar still," Desalination, vol. 245, no. 13, pp. 246-265, 2009.

[9] K. Sampathkumar, T. Arjunan, P. Pitchandi, and P. Senthilkumar, "Active solar distillation-a detailed review," Renewable and Sustainable Energy Reviews, vol. 14, no. 6, pp. 1503-1526, 2010.

[10] V. Dimri, B. Sarkar, U. Singh, and G. Tiwari, "Effect of condensing cover material on yield of an active solar still: an experimental validation," Desalination, vol. 227, no. 1-3, pp. 178-189, 2008. 